\title{
Ethnoecological Survey of Underutilized Plant Diversity of Hamirpur District, Himachal Pradesh, India: An Edibility Assessment
}

\author{
Ram Chand, Anand Narain Singh ${ }^{*}$ C. Nirmala
}

Department of Botany, Panjab University, India

Copyright $\bigcirc 2017$ by authors, all rights reserved. Authors agree that this article remains permanently open access under the terms of the Creative Commons Attribution License 4.0 International License

\begin{abstract}
The present study was conducted to explore diversity and status of underutilized wild edible plants of Hamirpur district, Himachal Pradesh, India. Total 85 plant species of 66 genera belonging to 39 families under various life forms and origins were recorded; in which, 30 species as trees ( 25 native and 5 exotic), 11 shrubs (6 native and 5 exotic), 28 herbs (10 native and 18 exotic) and remaining 16 were creepers (12 native and 4 exotic). Edibility index (EI) showed $100 \%$ score of edibility by two species, while $80 \%$ was shown by 11 species, $40 \%$ by 3 species and remaining 69 species showed 10 to $30 \%$ only. Across family, maximum utilization efficiency (10 score) was found only in 2 families (Apiaceae and Violaceae), while 5 families (Chenopodiaceae, Commelinaceae, Liliaceae, Oxalidaceae and Portulaceae) have shown 8 and one family (Amaranthaceae) exhibited 5.8 score followed by 31 other families. In this study, it was observed that consumption mode of various plant parts were either fresh or cooked. In conclusion, present study may help in alleviating food scarcity, malnutrition and can be employed further as commercial crop plants for the welfare of human in developing countries.
\end{abstract}

Keywords Wild Edible Plant, Utilization Efficiency, Edibility Index, Consumption Mode, Traditional Knowledge

\section{Introduction}

Plants have been playing an important vital role in human society. Evidently, about 40k to 100k species of plants have been used regularly in different geographical region for food, fodder, medicine, fiber, fuel, oil, timber, cultural and industrial purposes etc. ([1], [2], [3]).There are over 20,000 edible species as wild plants in the world [4], until now only 30 (thirty) plants are used to meet the $90 \%$ world's food requirement ([5], [4]). Only few staple crops such as wheat, rice, maize and millet produces majority of food supply globally. Due to global rise in population with projected nine billion people by 2050 [6], one of the greatest challenges facing world today is to feed the teeming population.

Several countries including India suffer famines and food shortage, although they have the potential to produce adequate nutritional food for the population [7]. It will be difficult to meet daily global requirement calories by three or four staple crops. Therefore, broader range of plant species which are neglected or underutilized plants can contribute significantly to meet the requirement of healthy diet, appropriate food and energy requirements of each human being. These underutilized plants can be a good alternative source of food demand and contribute toward food security, nutrition, health and income generation [8].

Information regarding these plants is not properly documented from certain remote areas especially from hilly regions. Some plant species have regional importance grown by local people and some plants grow wild and are used by local people to meet their energy need and to get healthy food. Some such plants like Buckwheat (Fagopyrum esculentum) and Raggi (Eleusine coracana) with high nutritive content have been introduced as substitute of staple crops in some region but still remain unexplored or underutilized ([9], [10], [11]). Similarly, Bamboo shoots are underutilized as food but now are being projected as a health food ([12], [13]). However, a large proportion of wild edible plants are used for eating and for medicinal purposes in different regions of India but use of wild plants without sufficient knowledge may give rise to adverse effects [14]. Though ethno-botanical studies are increasing, but traditional knowledge of many plants has not been documented yet properly [15]. Although, wild edible plants is a main component of underutilized plants, in various life forms, while distributed in different ecosystems. However, there are about 1532 edible wild food species reported in India [16]; in which, over 675 species are known from Indian Himalayan region [3], and it is estimated that 800 species are 
consumed as wild edible plants chiefly by tribal people [17].

Himachal Pradesh is a hilly state having vast diversity of plants and also known as store house of traditional, locally adopted indigenous and naturalized species (might be exotic) that providing fruits, vegetable and medicines etc. are lesser known or unexploited. These plants might have high nutritive potential and could be of great potential towards solving problems of food security. Evidently, some studies have been conducted and provided ample information regarding edibility of wild species. For example, Chauhan et al., [18] documented 34 tree plants of 14 families from Pabbar valley district Shimla; Rana et al., [19], studied 67 species and reported 22 species used as food from remote and tribal areas of Panghi valley from Chamba district; whereas, Sharma et al., [20] reported 43 wild edible plants from Murari Devi and surrounding area of Mandi district. Similar studies also been reported from other parts of Himachal Pradesh ([21], [22], [23]).

However, information regarding the ethno-ecological purpose of the plants has not been documented extensively in Himachal Pradesh as whole and no work has been conducted in the selected study area Hamirpur. Hence there is a need to document the folk and traditional knowledge of edible underutilized plants used by local people. So present study was conducted to estimate diversity and status of edible underutilized and wild plants and its edibility, utilization efficiency and consumption mode with following objectives: a) to estimate the diversity, utilization efficiency and consumption mode of collected plant species, b) to explore the traditional information of plants used for edible purposes by local people and c) to find out the probability of proper utilization of these underutilized plants for the food and pharmaceutical industry. We consider collected all these underutilized plants as "edible wild plants" because they are directly produced from pristine nature and growing without intentional cultivation which includes prevalently being indigenous and naturalized species. Also, admitted with two assumptions, 1) that wild edible plants are indeed having with multiple uses from several components (root, stem, leaf etc.) for food and medicinal purposes if collecting at any appropriate stage of growth and properly prepared; 2) we did not pay any attention on negative role of the plant species listed as exotic species excepts its edibility and uses. Although, many floristic assessments from various parts of India conducted and reported that about 30-40\% herbaceous flora of the total are not indigenous in nature while they are from other place of origin. Many of them from exotic species have been naturalized and pay good ecosystem services to the local people.

\section{Methods}

\subsection{Study Sites}

The study area comprises of several villages of Hamirpur district located at the south western part of Himachal Pradesh.
It lies between $76^{0}-17^{\prime}-50^{\prime \prime}$ to $76^{0}-43^{\prime}-42^{\prime \prime}$ east longitudes and $31^{\circ}-24^{\prime}-48^{\prime \prime}$ to $31^{\circ}-53^{\prime}-35^{\prime \prime}$ north latitudes (Figure 1). It is covered by lower Himalayas; the elevation varies from 400 to 1100 meters. The district is covering an area of 1118 $\mathrm{km}^{2}$ of total geographical region of the state and it is bounded in the north by river Beas which separates from Kangra district and in the east, Bakar and Seer Khads separates from Mandi district, whereas in the south, it is bounded by Bilaspur district and in the west by Una district. The climate is mainly sub-tropical and is not much hilly being closer to the plains. Annual average rainfall is about $1520 \mathrm{~mm}$, where maximum rain fall occurs in the month of July to September and minimum in the months of April to June. In this region, demographic structures are mixed tribal communities along with other different castes such as Brahmins, Rajputs, Kolis, Khatti, and Dumanas etc. However, tribal population in this region is mainly Gujjar and Gaddis at the interior parts migrated from Chamba and Lahul Sapiti.

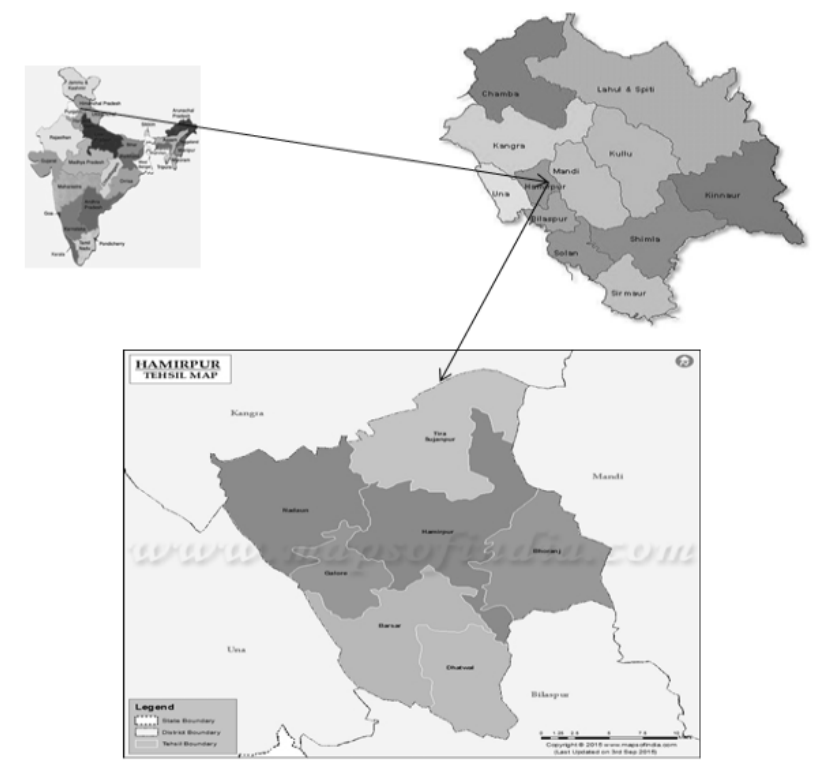

Figure 1. Location of the selected study sites at Hamirpur district, Himachal Pradesh.

\subsection{Experimental Design, Sampling and Monitoring Methods}

Field study was conducted at selected sites of Hamirpur district (sub-divisional area of the district known as tehsil), Himachal Pradesh during the period of 2013 to 2015. Therefore, three tehsils named as: Nadaun, Barsar and Hamirpur, in each tehsil, 10 Panchayat (includes of several villages) were selected for study. At each Panchayat, five households were taken from each village as informants for inquiring and collecting information. Selection of informants at each village at Panchayat was made by following criteria: 1) each household should have knowledge about plants, 2) household must have awareness about use and application of local plants for edible as well as for medicinal purposes, 3 ) 
household must have used to local climate and environment 4) household must be resident of the selected area. Therefore, in total, 300 informants (100 informants per tehsil) were interviewed across sites.

Field survey and sampling was carried out to collect information regarding status, utilization and edible sense of wild plants with involvement of local informants from each selected number of villages ( 2 villages of each Panchayat). Therefore, collected information was monitored at each selected Panchayat to take proper interaction and interview in respect to proper utilization of wild plants for food purposes.

In order to get satisfactory survey, a questionnaire was prepared pertaining to several required aspects and it was circulated among the informants. The information was collected in the native dialect (Pahari- close to Hindi), and recorded on the native names of the plants and their various uses for which plants are employed or used by the local people. The plant and its utility were assessed on the basis of consensus and confirmation by agreement of number of informants when data were pooled and subjected for statistical calculation.

Voucher number of each recorded plant was indexed with an established code (13E001 to 13E0085) and specimens of all the plants reported to be edible were collected, and herbarium was prepared. Each plant specimen was also visually documented with digital camera (Nikon P-500 model) for future prospect and proper records. Collected plant specimens were commonly available and known by the local people were identified in the field; while unidentified plants were identified at the herbarium center of Department of Botany, Panjab University Chandigarh (one of key herbarium Centre in country), India for proper identification.

In this paper, we simply reported edibility of total collected plant species based upon collected information across various informants and sites, also explained their mode of consumption, utilization efficiency and the number of uses as species-wise, family-wise of each species is discussed. Informant consensus data was subjected to statistical analyses, for example, to understand role of informants and variability of sites were analyzed for analyses of variance by SPSS version 14.0.

\subsection{Edibility Index}

In this study, a component wise use was developed and given weighted scale values according to the use size of each component; it was categorized into ten components of each plant having utilization for edible purposes, therefore, each component is allocated with 10 percent weighted score to calculate the values for this index.

In addition, summation of each aerial component use of each edible plant such as leaf, flower, fruit, seed, stem, resin, gum and shoot has been categorized as a whole aerial plant parts (WAP) and further summation of total component use as whole plant (WP) is given as 100 percent weighted score.
Therefore EI (\%) of each component will have minimum score as 10 percent and maximum will be 100 percent, therefore, EI of each species is calculated as:

$$
E I(\%)=\frac{P U i}{W P U i} \times 100
$$

Where, EI $=$ Edibility index, $\mathrm{PUi}=$ parts used of $i^{\text {th }}$ species, $\mathrm{WPU}_{\mathrm{i}}=$ whole plant use of $i^{\text {th }}$ species.

\subsection{Utilization Efficiency (UE)}

Utilization efficiency (UE) is calculated on the basis of edible plant parts used of all species recorded within each family, hence, summation of total number of plant parts used of all species divided by total number of plant species of each family:

$$
U E=\sum_{i}^{n} \frac{T N P P S U}{T N P S}
$$

Where, UE = Utilization Efficiency, TNPPsU $=$ Total number of plant parts used of all species and TNPS $=$ Total number of plant species within family.

\section{Results}

\subsection{Status of Edible Plants Diversity}

In this study, total 85 plant species of 66 genera belonging to 39 families were collected as edible plants from selected sites at Hamirpur district, Himachal Pradesh (Table 1). Vegetation composition at selected sites were distributed in various life forms; of which, 30 species areas trees $(35.3 \%$, 25 native and 5 exotic), 11 shrubs $(12.9 \%, 6$ native and 5 exotic), 28 herbs (33.0\%, 10 native and 18 exotic) and remaining 16 as creepers $(18.8 \%, 12$ native and 4 exotic) are presented in Figure. 2. However, significant number of the recorded edible plant species were trees followed by herbs (most dominant life form), while other growth forms of the plant like shrubs and creepers were sparsely distributed (Figure 2).

In order to get more clarity and status of edible plant species, a family-wise distribution of collected plants is assessed and presented in Figure 3. Out of all plant species, maximum number of species were found from family Moraceae (11) followed by Cucurbitaceae (8), Fabaceae (6), Amaranthaceae and Rosaceae (5 each); whereas, 14 families like Araceae, Asparagaceae, Asteraceae, Boraginaceae, Chenopodiaceae, Combretaceae, Dioscoreaceae, Euphorbiaceae, Lythraceae, Myrtaceae, Oxalidaceae, Rhamnaceae, Rutaceae and Solanaceae were sparsely found (2 to 4 species), while remaining 20 families had minimally one species each (Figure 3). 


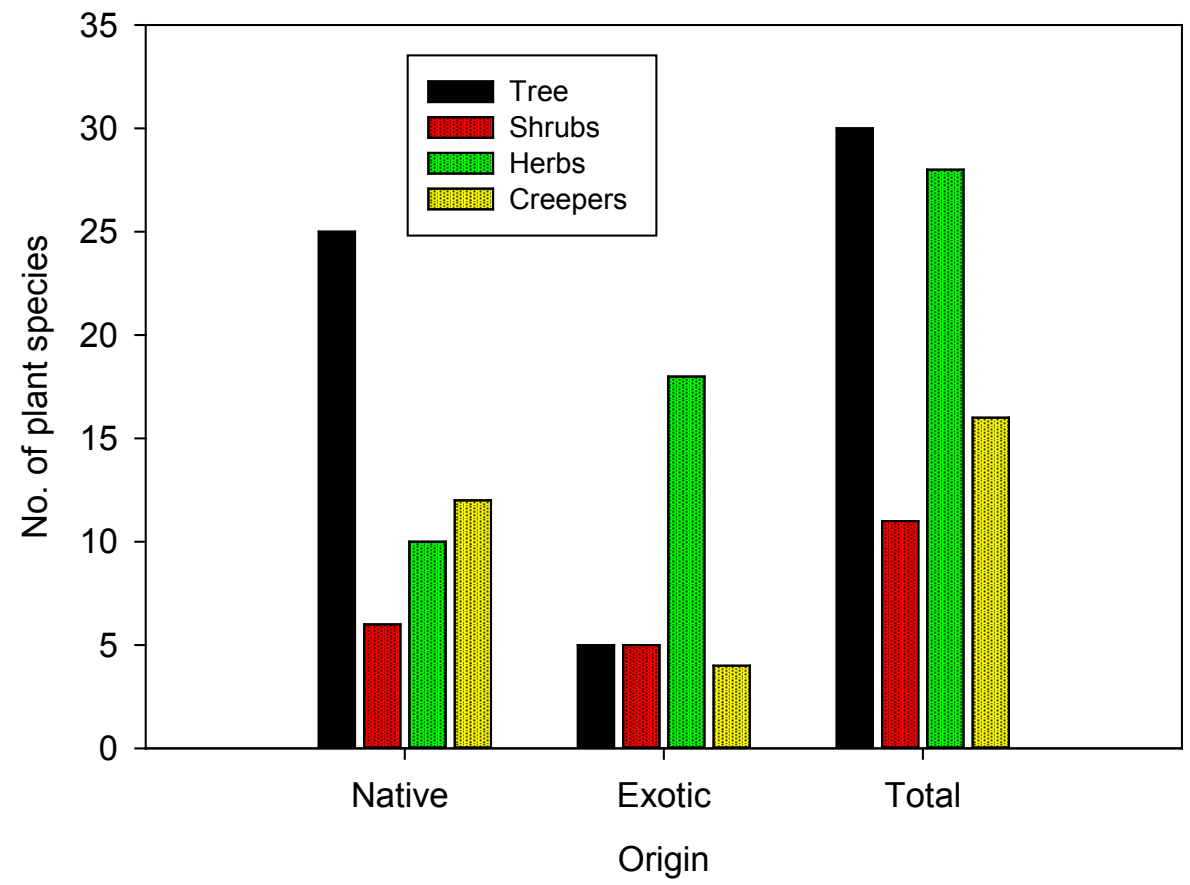

Figure 2. Total number of plant species recorded in various Life-forms at research site of Hamirpur district, Himachal Pradesh.

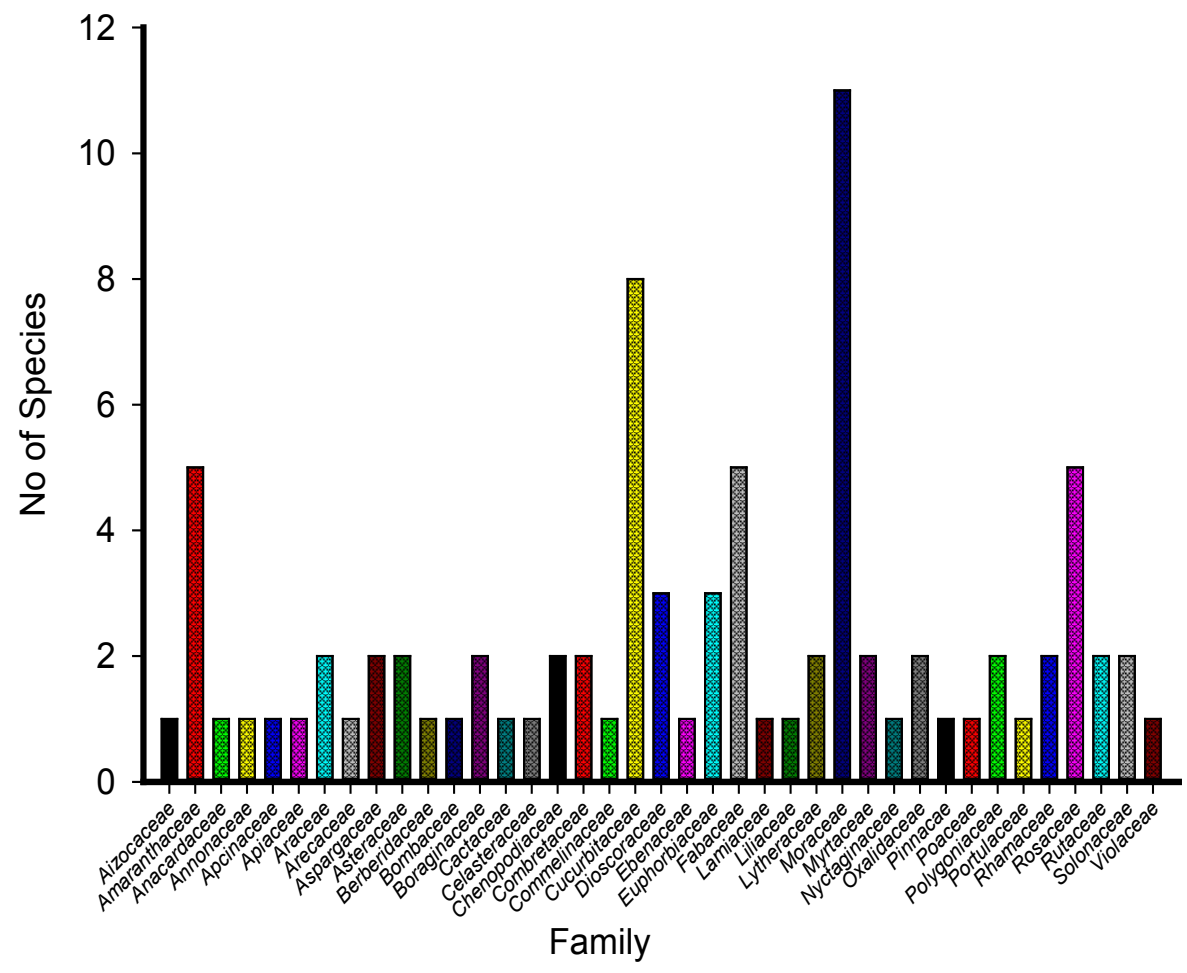

Figure 3. Family-wise distribution of recorded plant species of Hamirpur district, Himachal Pradesh 
Table 1. List of recorded edible plant species from selected research sites at Hamirpur district, Himachal Pradesh

\begin{tabular}{|c|c|c|c|c|c|c|}
\hline S. N./V.N. & $\begin{array}{l}\text { Scientific name of } \\
\text { plant species }\end{array}$ & Local Name & Vernacular Name (Hindi \& English) & Family & $\mathrm{O} / \mathrm{LF}$ & Uses \\
\hline $13 \mathrm{E} 001$ & $\begin{array}{l}\text { Aegle marmelos }(\mathrm{L} .) \\
\text { Correa }\end{array}$ & Bil/Bael & $\begin{array}{l}\text { H: Belpatthar Ka paid/ Belada mara/ Bilva/ Bael } \\
\text { E: Golden apple/ Stone apple }\end{array}$ & Rutaceae & $\mathrm{N} / \mathrm{T}$ & $\begin{array}{c}\text { Ripened fruits are used to prepare Murrubba, Jam, Juice. Also, used as } \\
\text { cooling laxative and aromatic to prepare Sharbat. Leaves are cooked } \\
\text { in tea and young shoots used as salad. Ritual importance, offering } \\
\text { young leaf to lord Shiva in worship. }\end{array}$ \\
\hline $13 \mathrm{E} 002$ & $\begin{array}{l}\text { Artocarpus lakoocha } \\
\text { Buch.-Ham. }\end{array}$ & Tiau & $\begin{array}{l}\text { H: Lakoocha/ Lakooch Barhal/ Dahu/ Dahua E: } \\
\text { Monkey Fruit }\end{array}$ & Moraceae & $\mathrm{N} / \mathrm{T}$ & $\begin{array}{l}\text { Young and mature fruits used in pickle. Flower and its buds cooked as } \\
\text { vegetable. }\end{array}$ \\
\hline 3 & Annona squamosa L. & Sheetalphal & H: Sheetaphal E:Custard apple & Annonaceae & $\mathrm{E} / \mathrm{T}$ & $\begin{array}{l}\text { Fruits are eaten after ripe. } \\
\end{array}$ \\
\hline 4 & $\begin{array}{l}\text { Bauhinia variegata } \\
\text { L. }\end{array}$ & Karaal & H:Kachnar E: Orchid Tree/ Variegated Bauhinia & Fabaceae & $\mathrm{N} / \mathrm{T}$ & $\begin{array}{c}\text { Flower and its buds are eaten as vegetable. Boiled flower buds used } \\
\text { with mustard oil as salads and raita. }\end{array}$ \\
\hline 5 & Bombax ceiba $\mathrm{L}$. & Simbal/Simmal & $\begin{array}{c}\text { H:Shalmali/ Semal E Silk Cotton Tree/ Kapok } \\
\text { Tree }\end{array}$ & Bombacaceae & $\mathrm{N} / \mathrm{T}$ & Flower buds cooked as vegetable. \\
\hline 6 & $\begin{array}{l}\text { Butea monosperma } \\
\text { (Lam.) Kuntze }\end{array}$ & Palah/palash & H: Palah/ Palash E: Parrot tree/ Flame of Forest & Fabaceae & $\mathrm{N} / \mathrm{T}$ & $\begin{array}{l}\text { Flowers are cooked as vegetable; its gum is used to prepare gond (kind } \\
\text { of latex) i.e. for pregnant women. Whereas, its decoction made in ball } \\
\text { shaped (laddoo) eaten during winter season. }\end{array}$ \\
\hline 7 & Cassia fistula $\mathrm{L}$. & Ali/Alis & H:Amaltaas E: Golden shower tree & Fabaceae & $\mathrm{N} / \mathrm{T}$ & $\begin{array}{c}\text { Young pods are used as pickles. Cut into small pieces, fried with } \\
\text { sarson oil, mixed with spices like turmeric, red-chilli and salt, then } \\
\text { kept it for } 15 \text { days for the use. Flowers are used to prepare raita and } \\
\text { gulukand. }\end{array}$ \\
\hline 8 & $\begin{array}{l}\text { Cordia dichotoma } \\
\text { G. Forst. }\end{array}$ & Lasura & H:Lasura E Indian Cherry/Clammy cherry & Boraginaceae & $\mathrm{E} / \mathrm{T}$ & $\begin{array}{l}\text { The leaves are cooked as vegetable whereas ripe fruits eaten directly } \\
\text { and unripe fruits are used to prepare pickle and vegetable with unripe } \\
\text { mango fruit slices. This preparation is very tasty and common among } \\
\text { the local people. }\end{array}$ \\
\hline 9 & $\begin{array}{l}\text { Cordia sinensis } \\
\quad \text { Lam. }\end{array}$ & Lasuri & H: Chhoti lasuree E: Small cordia & Boraginaceae & $\mathrm{E} / \mathrm{T}$ & $\begin{array}{l}\text { The leaves are cooked as vegetables, ripe fruits eaten directly and } \\
\text { unripe fruits are used to prepare pickle and vegetable with unripe } \\
\text { mango fruit slices. This preparation is very tasty and common among } \\
\text { the local people. }\end{array}$ \\
\hline 10 & $\begin{array}{l}\text { Diospyros } \\
\text { melanoxylon Roxb. }\end{array}$ & Kinnu & $\begin{array}{l}\text { H: Tendu, Timburni E: Coromandel Ebony or } \\
\text { East Indian Ebony }\end{array}$ & Ebenaceae & $\mathrm{N} / \mathrm{T}$ & Fruits are eaten after ripe. \\
\hline 11 & $\begin{array}{l}\text { Ficus auriculata } \\
\text { Lour. }\end{array}$ & $\begin{array}{l}\text { Tiyamal/Traymbal } \\
\text { u }\end{array}$ & $\begin{array}{c}\text { H: Timla/ Tirmal/Fagoora,/Tiaambal E: Elephant } \\
\text { Ear Fig }\end{array}$ & Moraceae & $\mathrm{N} / \mathrm{T}$ & Ripe figs are eaten while unripe fruits used as vegetable. \\
\hline $13 \mathrm{E} 012$ & $\begin{array}{l}\text { Ficus benghalensis } \\
\mathrm{L} .\end{array}$ & Bar/Bargadh & H: Barh, Bargad E: Banyan & Moraceae & $\mathrm{N} / \mathrm{T}$ & $\begin{array}{l}\text { Fruits are eaten. In the past, when resources were limited then fruits } \\
\text { were grounded into flour to make chapati. }\end{array}$ \\
\hline 13 & Ficus carica $\mathrm{L}$. & Dogla & Hindi - Anjeer E: Fig & Moraceae & $\mathrm{E} / \mathrm{T}$ & Ripened fruits eaten as fresh and unripe fruits are cooked as vegetable. \\
\hline 14 & $\begin{array}{l}\text { Ficus glomerata } \\
\text { Roxb. }\end{array}$ & Umbar/Ummar & H: Goolar E: Cluster fig & Moraceae & $\mathrm{N} / \mathrm{T}$ & $\begin{array}{l}\text { Ripened fruits are eaten directly but unripe fruits are cooked as } \\
\text { vegetable. }\end{array}$ \\
\hline 15 & Ficus hispida L. f. & Dangur & $\begin{array}{l}\text { H: Gobla, Kagsha, Kala Umbar, Katgularia, E: } \\
\text { Hairy Fig/ Devil Fig }\end{array}$ & Moraceae & $\mathrm{N} / \mathrm{T}$ & Unripe fruits are seldom used as vegetable. \\
\hline 16 & $\begin{array}{l}\text { Ficus palmata } \\
\text { Forssk. }\end{array}$ & Anjeer & H: Abjiri/Bedu E: Wild Fig & Moraceae & $\mathrm{N} / \mathrm{T}$ & Ripe fruits are eaten, also, used in sweets preparation. \\
\hline
\end{tabular}




\begin{tabular}{|c|c|c|c|c|c|c|}
\hline 17 & Ficus religiosa $\mathrm{L}$. & Pipal/Piplu & H: Peepal E: Sacred Fig/Holly tree & Moraceae & $\mathrm{N} / \mathrm{T}$ & Same use as of Ficus benghalensis (Bargad). \\
\hline 18 & $\begin{array}{l}\text { Ficus semicordata } \\
\text { Buch.-Ham.ex. Sm. }\end{array}$ & Khain/ Khainu & H: Khaina/ Khunia, E: Drooping Fig & Moraceae & $\mathrm{N} / \mathrm{T}$ & Ripe fruits are eaten. \\
\hline 19 & $\begin{array}{l}\text { Flacourtia indica } \\
\text { (Burm. f.) Merr. }\end{array}$ & Kaangu & $\begin{array}{l}\text { H: Kangu E: Indian Plum/Flacourtia/Governor's } \\
\text { Plum }\end{array}$ & Moraceae & $\mathrm{N} / \mathrm{T}$ & Ripened fruits are directly eatable and unripe used for making pickle. \\
\hline 20 & Morus alba $\mathrm{L}$. & Tut/ Shahtut & H: Shahtoot E: Mul berry/ Silkworm Mulberry & Moraceae & $\mathrm{E} / \mathrm{T}$ & Young leaves are cooked as vegetable; ripe fruits are eaten directly. \\
\hline 21 & $\begin{array}{l}\text { Phoenix sylvestris } \\
\text { (L.) Roxb. }\end{array}$ & Khajoor & $\begin{array}{l}\text { H: Khajoor E: Silver Date Palm or Sugar Date } \\
\text { Palm }\end{array}$ & Arecaceae & $\mathrm{N} / \mathrm{T}$ & Ripe Fruits are eatable directly. \\
\hline 22 & $\begin{array}{l}\text { Phyllanthus emblica } \\
\text { L. }\end{array}$ & Aambla & $\begin{array}{l}\text { H: Amla/ Aambla E: Indian gooseberry/ Malacca } \\
\text { Tree }\end{array}$ & Euphorbiaceae & $\mathrm{N} / \mathrm{T}$ & $\begin{array}{l}\text { Good medicinal plant; fruits are commonly used for pickle. Also } \\
\text { eaten as raw or cooked for various dishes. A sweet pickle and } \\
\text { murrubba is commonly used in northern part of India. Also prepare } \\
\text { sugar candy. In this region, local people used it as sweet sour sausage } \\
\text { and fruits either ripe or unripe are used in daily life. }\end{array}$ \\
\hline 23 & $\begin{array}{l}\text { Pinus roxburghii } \\
\text { Sarg. }\end{array}$ & $\begin{array}{l}\text { Chir/Chil/ } \\
\text { Chalautu }\end{array}$ & H: Chirh E: Chir Pine, Three Leaved Pine & Pinaceae & $\mathrm{N} / \mathrm{T}$ & Seeds are eaten as raw as well as roasted. \\
\hline 24 & $\begin{array}{l}\text { Pyrus pashia } \\
\text { Buch.-Ham. }\end{array}$ & Kainth & H: Mehal/ Mole/Kainath/ E: Indian wild pear & Rosaceae & $\mathrm{N} / \mathrm{T}$ & Ripe fruits are eaten. \\
\hline 25 & $\begin{array}{l}\text { Spondias pinnata } \\
\text { (L.f.) } \\
\text { Kurz. }\end{array}$ & $\begin{array}{l}\text { Ambara, Buara, } \\
\text { Jangli Aam }\end{array}$ & $\begin{array}{c}\text { H: Ambara/ Ambari/ Amra E: Wild Mango/Indian } \\
\text { hog plum }\end{array}$ & Anacardiaceae & $\mathrm{N} / \mathrm{T}$ & $\begin{array}{l}\text { This is a valuable tree for food and medicine. Its unripe fruits are } \\
\text { acidic and eaten fresh as well as cooked as pickle and vegetable, } \\
\text { Chuttaney etc. Sometime Juice or Jam of ripped fruits is also prepared. } \\
\text { Ripe or unripe fruits are cut into small pieces; dried, powdered and } \\
\text { preserved form is useful in off season. }\end{array}$ \\
\hline 13E026 & $\begin{array}{l}\text { Syzygium } \\
\text { jambolanum (Lam.) } \\
\text { DC. }\end{array}$ & Pataman/Jamoa & H: Jamoon E: Black Plum & Myrtaceae & $\mathrm{N} / \mathrm{T}$ & Fleshy ripened fruits are eaten directly. \\
\hline 27 & $\begin{array}{l}\text { Syzygium cuminii } \\
\text { (L.) Skeel. }\end{array}$ & Jamman, Jammun & H: Jamun E: Java plum & Myrtaceae & $\mathrm{N} / \mathrm{T}$ & $\begin{array}{l}\text { Ripe fruits are eaten, but uses have declined in villages and the fruits } \\
\text { are being sold in the cities. }\end{array}$ \\
\hline 28 & $\begin{array}{l}\text { Terminalia bellirica } \\
\text { (Gaertn.) Roxb. }\end{array}$ & Bahera & H: Bahera E: Bastard myrobalan & Combretaceae & $\mathrm{N} / \mathrm{T}$ & Fruits are used to prepare triphala and pickles \\
\hline 29 & $\begin{array}{l}\text { Terminalia chebula } \\
\text { Retz. }\end{array}$ & Harar & H: Hara / Harada, E: Chebulic myrobalan & Combretaceae & $\mathrm{N} / \mathrm{T}$ & Fruits are used to prepare triphala and pickles \\
\hline 30 & $\begin{array}{l}\text { Ziziphus jujuba Mill. } \\
\text { Gard. }\end{array}$ & Ber & H: Ber E: Indian Jujube/ Indian Plum & Rhamnaceae & $\mathrm{N} / \mathrm{T}$ & Ripe fruits are eaten directly. \\
\hline 31 & Agave americana $\mathrm{L}$. & Kayora & $\begin{array}{l}\text { H: Gwarpatha E: Century plant/ } \\
\text { maguey/American aloe }\end{array}$ & Asparagaceae & $\mathrm{E} / \mathrm{S}$ & $\begin{array}{l}\text { Young shoot is peeled off and cut into small pieces, boiled in water, } \\
\text { squeezed and then fried with oil to prepare a dish along with using } \\
\text { certain common Indian spices. }\end{array}$ \\
\hline 32 & $\begin{array}{l}\text { Berberis asiatica } \\
\text { Roxb. Ex DC. }\end{array}$ & Kashmal & $\begin{array}{c}\text { H: Chitra/Chotra E: Indian Barberry, Tree } \\
\text { Turmeric }\end{array}$ & Berberidaceae & $\mathrm{N} / \mathrm{S}$ & $\begin{array}{l}\text { The fruit is eaten as raw or dry and used as raisins. This species is said } \\
\text { to make the best Indian raisin. Ripe fruits are juicy with pleasantly } \\
\text { acid flavour. }\end{array}$ \\
\hline 33 & Carissa spinarum $\mathrm{L}$. & Garuna, Gurnu & H: Karonda E :Conkerberry /Bush plum & Apocynaceae & $\mathrm{N} / \mathrm{S}$ & $\begin{array}{l}\text { Fruits are eaten. Young shoots are peeled off and eaten by children. } \\
\text { Raw fruits are used in pickle. }\end{array}$ \\
\hline 34 & $\begin{array}{l}\text { Euphorbia royleana } \\
\text { Boiss. }\end{array}$ & Chhun, Thoar & H: Thoar E: Royle's Spurge & Euphorbiaceae & $\mathrm{E} / \mathrm{S}$ & $\begin{array}{l}\text { Young shoots of the stem is peeled off, cut into pieces and boiled in } \\
\text { water, squeezed, then fried and cooked with vegetable. }\end{array}$ \\
\hline
\end{tabular}




\begin{tabular}{|c|c|c|c|c|c|c|}
\hline 35 & $\begin{array}{l}\text { Murraya koenigii L. } \\
\text { Spreng. }\end{array}$ & Gandhala & H: Mitha neem, Kurry patta E: Curry leaf-tree & Rutaceae & $\mathrm{N} / \mathrm{S}$ & Leaves are used as condiments as well as curry preparation. \\
\hline 36 & $\begin{array}{l}\text { Opuntia dillenii } \\
\text { Haw. }\end{array}$ & Pahari choon & H: Nagphani E:Prickly Pear & Cactaceae & $\mathrm{E} / \mathrm{S}$ & Ripe fruits are eaten after removing spines. \\
\hline 37 & Punica granatum L. & Daran & H: Jangali Anar E: Dwarf Pomegranate & Lythraceae & $\mathrm{N} / \mathrm{S}$ & Ripe fruit is eaten and also prepared as digestive ball (aanardana). \\
\hline 38 & $\begin{array}{l}\text { Rosa moschata } \\
\text { Herrm. }\end{array}$ & Jangli Gulaab & H: Ban gulab E: Musk Rose & Rosaceae & $\mathrm{E} / \mathrm{S}$ & Flowers are used to prepare gulukand. \\
\hline 39 & Rubus ellipticus $\mathrm{Sm}$. & Aakha & H: Lalanchu/ Hinsa E: Roadleafed bramble & Rosaceae & $\mathrm{N} / \mathrm{S}$ & Ripened fruits are eaten. \\
\hline 40 & $\begin{array}{l}\text { Woodfordia } \\
\text { fructicosa } \mathrm{L} \text {. }\end{array}$ & Dhaun & H: Dhawai E: Fire Flame Bush & Lythraceae & $\mathrm{N} / \mathrm{S}$ & $\begin{array}{l}\text { Flowers have sweet nectar which is sucked by the people and these } \\
\text { may be used as vegetable. }\end{array}$ \\
\hline 41 & $\begin{array}{l}\text { Ziziphus mauritiana } \\
\text { lamk. }\end{array}$ & Malair & H: Ban Ber E: Indian Jujube, Chinese Date & Rhamnaceae & $\mathrm{E} / \mathrm{S}$ & Fruits are eaten. \\
\hline 13E0042 & $\begin{array}{l}\text { Achyranthus aspera } \\
\text { L. }\end{array}$ & Puthkanda & H: Chirchita E: Prickly Chaff Flower & Amaranthaceae & $\mathrm{N} / \mathrm{H}$ & $\begin{array}{c}\text { Husk free seeds used for halwa preparation with milk. In the past when } \\
\text { the source was limited seed flour was used to prepare chapati. Halwa } \\
\text { prepared with seeds are highly nutritive and energetic. Generally, } \\
\text { Hindu monk (naga) used before starting fast and meditation for } \\
\text { several days. }\end{array}$ \\
\hline 43 & $\begin{array}{l}\text { Colocasia esculenta } \\
\text { (L.) Schott }\end{array}$ & Jangli kachalu & H: Arvi, Ashukachu, E: Taro, Colocasia root & Araceae & $\mathrm{E} / \mathrm{H}$ & $\begin{array}{l}\text { Tubers used as vegetable. Leaves are also used as leafy vegetable. } \\
\text { Leaves are used for making some specific food menu in local culture. }\end{array}$ \\
\hline 44 & $\begin{array}{l}\text { Aloe vera (L.) } \\
\text { Burm.f. }\end{array}$ & Kuaar/Kunwar & H: Gheekumari E: Aloe vera & Asparagaceae & $\mathrm{E} / \mathrm{H}$ & $\begin{array}{c}\text { Ariel part is used to prepare vegetable, pickle and juice. Roots are also } \\
\text { used for herbal medicines in local culture. }\end{array}$ \\
\hline 45 & $\begin{array}{l}\text { Amaranthus } \\
\text { cruentus L. }\end{array}$ & Kalaunji /Seul & H: Chaulai E: Red Amaranthes & Amaranthaceae & $\mathrm{E} / \mathrm{H}$ & Cooked into vegetable and halwa \\
\hline 46 & $\begin{array}{l}\text { Amaranthus viridis } \\
\text { L. }\end{array}$ & Chaulai & $\mathrm{H}$ :Jangli Chaulai E: African amaranthes & Amaranthaceae & $\mathrm{E} / \mathrm{H}$ & Cooked as leafy vegetable. \\
\hline 47 & $\begin{array}{l}\text { Amaranthus } \\
\text { spinosus } \mathrm{L} \text {. }\end{array}$ & Chaulai & H: Kanta chaulai E:Prickly Amaranth, Edlebur & Amaranthaceae & $\mathrm{E} / \mathrm{H}$ & $\begin{array}{l}\text { Leaves or young shoots are cooked as vegetable Leaves are also used } \\
\text { to prepare prantha. }\end{array}$ \\
\hline 48 & $\begin{array}{c}\text { Amorphophallus } \\
\text { paeoniifolius } \\
\text { ( Dennst.) Nicolson, }\end{array}$ & Jamikand & Zimikand & Araceae & $\mathrm{N} / \mathrm{H}$ & Tubers are cooked as vegetable. Good source of iron. \\
\hline 49 & $\begin{array}{l}\text { Artemisia scoparia } \\
\text { Waldst. \& Kit. }\end{array}$ & Chaunkhra & H: Seeta Bani E: Redstem wormwood & Asteraceae & $\mathrm{E} / \mathrm{H}$ & $\begin{array}{l}\text { Leaves and inflorescence are used for flavouring dal curry, vegetable } \\
\text { and in pickles. }\end{array}$ \\
\hline 50 & $\begin{array}{l}\text { Asphodelus } \\
\text { tenuifolius Cav. }\end{array}$ & Piyaaji & H: Piyaaji E: Onion-Weed & Liliaceae & $\mathrm{N} / \mathrm{H}$ & $\begin{array}{l}\text { Aerial parts used as vegetable and dry seeds in condiments. Seed flour } \\
\text { used in making chapatti. }\end{array}$ \\
\hline 51 & $\begin{array}{l}\text { Bambusa vulgaris } \\
\text { Schrad.ex. } \\
\text { J.C.Wendl. } \\
\end{array}$ & Bans, Bainj & H: Bans E: Common bamboo & Poaceae & $\mathrm{E} / \mathrm{H}$ & Young shoots are used to prepare vegetable and pickle. \\
\hline 52 & $\begin{array}{l}\text { Boerhavia diffusa } \\
\text { (L.) nom. Cons. }\end{array}$ & Utshat & H: Punarnava E: Hogweed & Nyctaginaceae & $\mathrm{N} / \mathrm{H}$ & $\begin{array}{l}\text { The leaves are cooked as vegetable. Also used with other edible } \\
\text { vegetables to prepare a mixed vegetable. }\end{array}$ \\
\hline 53 & $\begin{array}{l}\text { Centella asiatica } \\
\text { (L.) Urban }\end{array}$ & Brahmi & $\begin{array}{l}\text { H: Brahma manduk E: Indian Pennywort/ } \\
\text { Coinwort/Asiatic coinwort }\end{array}$ & Apiaceae & $\mathrm{N} / \mathrm{H}$ & $\begin{array}{l}\text { Whole plant is used to prepare a juice for enhancing memory power. } \\
\text { Raw leaf is eaten freshly. }\end{array}$ \\
\hline 54 & $\begin{array}{l}\text { Chenopodium } \\
\text { giganteum D. Don. }\end{array}$ & Bara bathu & H: Bathu E: Tree spinac & Chenopodiaceae & $\mathrm{N} / \mathrm{H}$ & Leaves cooked as vegetable or local dish sag. \\
\hline
\end{tabular}




\begin{tabular}{|c|c|c|c|c|c|c|}
\hline 55 & $\begin{array}{l}\text { Chenopodium album } \\
\text { L. }\end{array}$ & Kunna/Bathu & H: Bathua or Bathuwa E: Goosefoot & Chenopodiaceae & $\mathrm{E} / \mathrm{H}$ & $\begin{array}{l}\text { Leaves or young shoots are cooked as vegetable ie sag in a local } \\
\text { preparation. Leaves are also used to prepare chapati and parantha. }\end{array}$ \\
\hline 56 & $\begin{array}{c}\text { Commelina } \\
\text { benghalensis } \mathrm{L} .\end{array}$ & Chhura & H: Kanchara E: Day Flower/ Dew Flower & Commelinaceae & $\mathrm{E} / \mathrm{H}$ & Leaves are cooked as vegetable. \\
\hline 57 & $\begin{array}{l}\text { Digera muricata L. } \\
\text { Mart. }\end{array}$ & Chaulai & H: Chaulai E: False Amaranthes & Amaranthaceae & $\mathrm{E} / \mathrm{H}$ & Young leaves cooked as vegetable and raiyta. \\
\hline 58 & $\begin{array}{l}\text { Euphorbia } \\
\text { heterophylla } \mathrm{L} \text {. }\end{array}$ & Dudhali & H: Bari Dhudi E: wild poinsettia & Euphorbiaceae & $\mathrm{E} / \mathrm{H}$ & Leaves are used as vegetable ( Bhujia). \\
\hline 59 & $\begin{array}{l}\text { Ocimum basilicum } \\
\mathrm{L} .\end{array}$ & Bashala & H: Basil/ Bhabri E: Sweet Basile & Lamiaceae & $\mathrm{N} / \mathrm{H}$ & Leaves are used for flavouring dal and traditional dishes. \\
\hline 60 & $\begin{array}{l}\text { Oxalis corniculata } \\
\text { L. }\end{array}$ & Khatti- Mithi & H: Teen Patia E: Yellow wood sorrel & Oxalidaceae & $\mathrm{E} / \mathrm{H}$ & Whole plant used to prepare chuttney. \\
\hline 61 & $\begin{array}{l}\text { Oxalis rubra } \\
\text { A.St.Hil. }\end{array}$ & Khatti Mithi & H: Teen Patia E: Window box Wood sorrel & Oxalidaceae & $\mathrm{E} / \mathrm{H}$ & Leaves and young shoots are used to prepare Vegetable and chuttney. \\
\hline 62 & $\begin{array}{l}\text { Physalis peruviana } \\
\text { L. }\end{array}$ & Dophalu & H: Dophali,Rasbhari E: Cape Gooseberry & Solanaceae & $\mathrm{E} / \mathrm{H}$ & Ripened fruits are eaten freshly. \\
\hline 63 & $\begin{array}{l}\text { Portulaca oleracea } \\
\text { L. }\end{array}$ & Naneria & $\begin{array}{l}\text { H: Khursa/Kulfa E: Common purslane/ } \\
\text { Verdolaga, }\end{array}$ & Portulacaceae & $\mathrm{N} / \mathrm{H}$ & $\begin{array}{l}\text { Whole plant is used to prepare vegetable and also prepared vegetable } \\
\text { with pulses of black gram. }\end{array}$ \\
\hline 64 & Fragaria vesca $\mathrm{L}$. & Van Aakha & H: Van aakha E: wild strawberry & Rosaceae & $\mathrm{E} / \mathrm{H}$ & Ripened fruits are eaten. \\
\hline 65 & $\begin{array}{l}\text { Rumex hastatus } \\
\text { D.Don. }\end{array}$ & Bari Khatti Buti & $\begin{array}{l}\text { H: Churki/Churka E: Arrow leaf Dock/Yellow } \\
\text { Sock }\end{array}$ & Polygonaceae & $\mathrm{E} / \mathrm{H}$ & Leaves and young shoots are used to prepare vegetable and chuttney. \\
\hline 66 & Solanum nigrum $\mathrm{L}$. & $\begin{array}{l}\text { Karyaun } \\
\text { miyaun/Jangli } \\
\text { Mirch }\end{array}$ & $\begin{array}{l}\text { H: Mokoi E: Black nightshade/ Black-berry } \\
\text { night shade }\end{array}$ & Solanaceae & $\mathrm{N} / \mathrm{H}$ & Ripened fruits are eaten and young leaves are cooked as vegetable. \\
\hline 67 & $\begin{array}{l}\text { Spilanthes acmella } \\
\text { L. }\end{array}$ & Akarkara/ Karkara & $\begin{array}{c}\text { H: Akarkar, Pipulka E: Toothache Plant/Para } \\
\text { cress }\end{array}$ & Asteraceae & $\mathrm{E} / \mathrm{H}$ & Flowers are eaten fresh. \\
\hline 68 & $\begin{array}{c}\text { Trianthema } \\
\text { portulacastrum } \mathrm{L} .\end{array}$ & Shanti & H: Shanti/ Safed punarnava & Aizoaceae & $\mathrm{E} / \mathrm{H}$ & Leaves are eaten as vegetable. \\
\hline 69 & $\begin{array}{l}\text { Viola serpense } \\
\text { Wall.ex. Roxb. }\end{array}$ & Banafsa/Banaksha & H: Banafsha E: Himalayan White Viola & Violaceae & $\mathrm{N} / \mathrm{H}$ & Flowers are used to prepare hot drinks like tea. \\
\hline 70 & $\begin{array}{l}\text { Bauhinia vahlii } \\
\text { Wight \& Arnott, } \\
\text { Prodr. }\end{array}$ & Taur & H: Malu/Jallaur/Jallur/ Mahul E: Maloo Creeper & Fabaceae & $\mathrm{N} / \mathrm{C}$ & $\begin{array}{l}\text { Roasted seeds are eaten by the local people in past and use has been } \\
\text { declined at present. The roasted seeds provide strength to body or act } \\
\text { as body tonic. }\end{array}$ \\
\hline 71 & $\begin{array}{l}\text { Celastrus } \\
\text { paniculatus } \\
\text { Willd }\end{array}$ & Sankheru & H: Malkanghni E: Black oil plant & Celastraceae & $\mathrm{N} / \mathrm{C}$ & $\begin{array}{c}\text { Oil extracted from mature seeds, also seeds and flowers used as } \\
\text { vegetable. }\end{array}$ \\
\hline 13E0072 & Coccinia grandis $\mathrm{L}$. & Kundru & H: Kunduru E: Ivy Gourd & Cucurbitaceae & $\mathrm{N} / \mathrm{C}$ & Fruits are used as vegetable. \\
\hline 73 & $\begin{array}{l}\text { Cucumis callosus } \\
\text { (Rottl.) Cogn. }\end{array}$ & Kachari/ Phutanu & H: Kachari E: Bitter Cucumber & Cucurbitaceae & - & $\begin{array}{l}\text { Ripe fruits are tasty eaten as raw and vegetable, fruit are digestive. In } \\
\text { off season dried fruits are used to prepare vegetable and chuttney. }\end{array}$ \\
\hline 74 & $\begin{array}{l}\text { Dioscorea bulbifera } \\
\text { L. }\end{array}$ & $\begin{array}{l}\text { Tararian/Jangli } \\
\text { Aloo }\end{array}$ & $\begin{array}{c}\text { H: Jangli Aloo, E: Aerial yam, Air potato, Wild } \\
\text { yam }\end{array}$ & Dioscoraceae & $\mathrm{N} / \mathrm{C}$ & $\begin{array}{l}\text { The tubers are edible after processing. After removing small rootlets, } \\
\text { the tubers are boiled in water, cooled down and skin is removed. Then } \\
\text { they are cut into small slices. These slices are put in container or } \\
\text { perforated basket; kept in stream of water to remove the acrid contents } \\
\text { of the tuber. Also, chips can be prepared. Used in form of vegetable. }\end{array}$ \\
\hline
\end{tabular}




\begin{tabular}{|c|c|c|c|c|c|c|}
\hline 75 & $\begin{array}{l}\text { Dioscorea deltoidea } \\
\text { Wall. ex Kunth. }\end{array}$ & Singhali-minghali & $\begin{array}{l}\text { H: Shingalimingali E: Dioscorea/ Elephant } \\
\text { foot/Yam }\end{array}$ & Dioscoraceae & $\mathrm{N} / \mathrm{C}$ & Tubers are used as vegetable as dry as well as curry. \\
\hline 76 & Dioscorea alata L. & Junglee aloo & E: Wild Yam/ Purple Yam & Dioscoraceae & $\mathrm{E} / \mathrm{C}$ & The tubers are edible after processing as vegetable. \\
\hline 77 & $\begin{array}{l}\text { Duchesnea indica } \\
\text { (Andrews) Focke }\end{array}$ & Van aakha & $\begin{array}{l}\text { H: Kiphaliya E: Indian strawberry/ Mock } \\
\text { strawberry }\end{array}$ & Rosaceae & $\mathrm{N} / \mathrm{C}$ & Fruits are eaten freshly. \\
\hline 78 & $\begin{array}{l}\text { Luffa operculata } \\
\text { Cogn. }\end{array}$ & Van tori & H: Jangli Tori E: Ridge cucumber & Cucurbitaceae & $\mathrm{N} / \mathrm{C}$ & Cooked as vegetable \\
\hline 79 & $\begin{array}{l}\text { Momordica dioica } \\
\text { Roxb. ex Willd }\end{array}$ & Kakora & $\begin{array}{c}\text { H: Meetha Karela / Kankeda/ Poraru E: Spine } \\
\text { Gourd }\end{array}$ & Cucurbitaceae & $\mathrm{E} / \mathrm{C}$ & Fruits used as vegetable \\
\hline 80 & $\begin{array}{c}\text { Melothria } \\
\text { heterophylla (Lour.) }\end{array}$ & $\begin{array}{c}\text { Bon Kaduri/Jangli } \\
\text { Kakri }\end{array}$ & $\begin{array}{c}\text { H: Kaduri } \\
\text { E:Creeping Cucumber }\end{array}$ & Cucurbitaceae & $\mathrm{N} / \mathrm{C}$ & Fruits are edible. \\
\hline 81 & $\begin{array}{l}\text { Mucuna pruriens } \\
\text { (L.) DC. Prodr. }\end{array}$ & Durangal & H: Kiwach E: Velvet bean/Cowitch & Fabaceae & $\mathrm{N} / \mathrm{C}$ & $\begin{array}{l}\text { Unripe pods are used to prepare vegetable and seeds are used for } \\
\text { preparing dal curry. }\end{array}$ \\
\hline 82 & $\begin{array}{l}\text { Pueraria montana } \\
\text { (Lour.) Merr. }\end{array}$ & Salod/ Banaar & H:kudzu E: Kudzu vine & Fabaceae & $\mathrm{N} / \mathrm{C}$ & Young tubers are eaten without any processing. \\
\hline 83 & $\begin{array}{l}\text { Sechium edule } \\
\text { (Jacq.) Sw. }\end{array}$ & Loku & H: Chayote E: Chow-Chow & Cucurbitaceae & $\mathrm{E} / \mathrm{C}$ & $\begin{array}{l}\text { Fruits are cooked as vegetable and sour fermented preparation as } \\
\text { salads. }\end{array}$ \\
\hline 84 & $\begin{array}{l}\text { Trichosanthes } \\
\text { cucumerina var. } \\
\text { cucumerina L. }\end{array}$ & Mais & H: Charchinda/Chichonda E: Round Gourd & Cucurbitaceae & $\mathrm{N} / \mathrm{C}$ & Fruits are cooked as vegetable. \\
\hline 85 & $\begin{array}{c}\text { Trichosanthes } \\
\text { cucumerina var. } \\
\text { anguina }(\text { L.) Haines }\end{array}$ & Pandol & H: Charchinda/Chichonda E: Snake Gourd & Cucurbitaceae & $\mathrm{N} / \mathrm{C}$ & Fruits used as vegetable. \\
\hline
\end{tabular}

$\mathrm{O}=$ origin, $\mathrm{LF}=$ Life form, $\mathrm{N}=$ Native, $\mathrm{E}=$ exotic, $\mathrm{T}=$ tree, $\mathrm{S}=$ shrub, $\mathrm{H}=$ herb, $\mathrm{C}=$ creeper, $\mathrm{H}=$ Hindi, $\mathrm{E}=$ English 


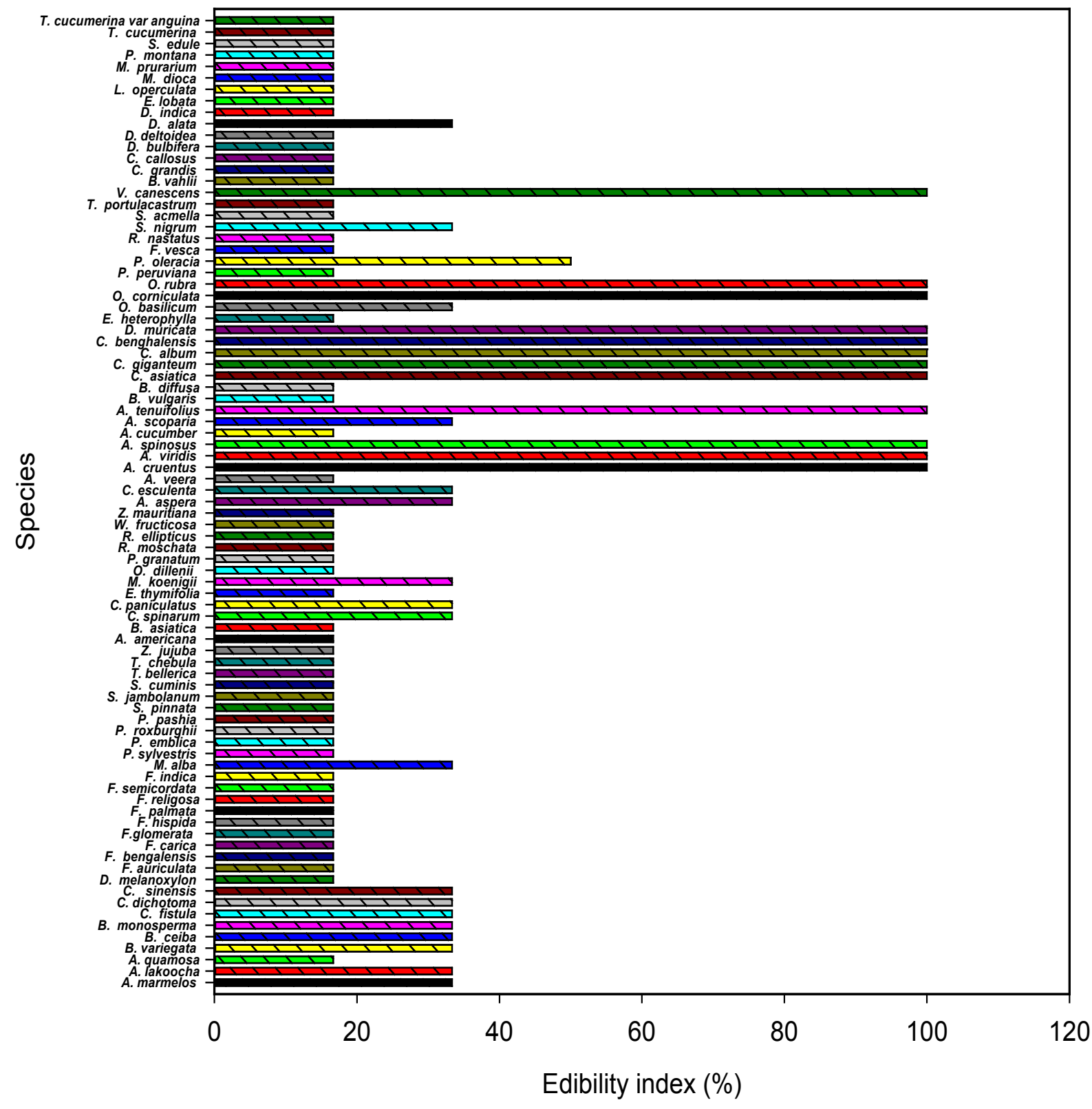

Figure 4. Edibility index (EI) of recorded plant species at research site. 


\subsection{Edibility Index}

Edibility index (EI) is one of the important indices which may give authentic information of edible sense of each species. EI was calculated and represented on a percent base weighted score. It has been developed on a component-wise use of each plant species which categorized into several components like root, stem, shoot, leaf and flower etc. Therefore, whole plant components were scored on a scale of 10-100 percent (Table 2). Out of total 85 species, 2 species showed hundred percent, eighty percent by 11 species, 40 percent by 3 species and remaining other species (69) showed 10-30 percent, respectively (Figure 4).

Table 2. Component wise use scale applied for calculation of edibility index of plant species at Hamirpur district, Himachal Pradesh. Each component use is allocated to the percent use of whole plant.

\begin{tabular}{ccc}
\hline S. & Component & Scale (\%) \\
No. & Flower and flower bud & 10.0 \\
1. & Fruit & 10.0 \\
2. & Seed & 10.0 \\
3. & Leaf & 10.0 \\
4. & included) & 10.0 \\
5. & Root and tuber & 10.0 \\
6. & Resin & 10.0 \\
7. & Gum & 10.0 \\
8. & Whole Aerial Part (WAP) & 80.0 \\
9. & Whole Plant (WP) & 100.0 \\
10. & & \\
\hline
\end{tabular}

\subsection{Utilization Efficiency}

Another index i.e. utilization efficiency (UE) per species per component is constructed in this study. UE was calculated on the basis of plant parts used of all recorded edible species within a family divided by total number of species recorded of each family. Values estimated for maximum utilization efficiency will be not more than 10 and not less than 1 for each family; therefore, recorded values of UE across family will be ranged from one to ten. Highest utilization efficiency (10) was found in two families: (Apiaceae and Violaceae) which indicates hundred percent utilization of all components for edible purposes, after that, corresponding values for collected members of five families (Chenopodiaceae, Commelinaceae, Liliaceae, Oxalidaceae and Portulaceae) have shown eight utilization efficiency which is significantly better than other remaining families (17) in which UE is only one which indicate at least one component is edible; while 14 families had more than one and less than four UE (Figure 5).Exceptionally, only one family (Amaranthaceae) had 5.8 UE score because the family members are herbaceous in nature and they had better utilization as leafy vegetable (Figure. 5).

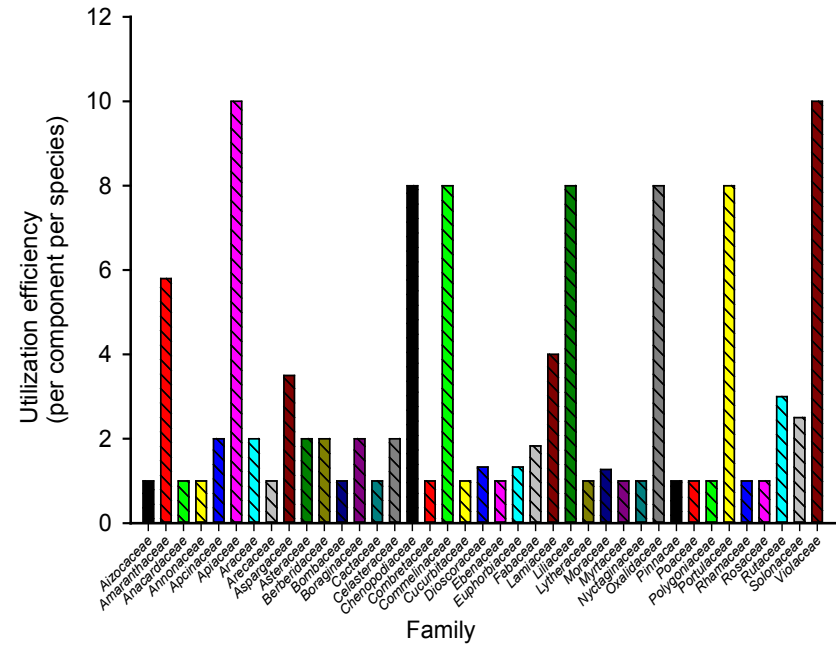

Figure 5. Family-wise utilization efficiency of recorded plants. Values presented in the graph are calculated based on family-wise total no. of plant parts used divided by total number of plant species of each family.

\subsection{Mode of Consumption}

Consumption of wild plants for the food purposes are perhaps storehouse of traditional knowledge acquired by local people. It was observed in this study that consumption mode of various plant parts were used as mainly as fresh or cooked form. Maximum numbers of wild plants as fruits are generally eaten raw when they ripe and usually unripe fruits, seeds, flowers and leaves are cooked as vegetables (Figure 6). Across component-wise use, highest number of edible species was used as fruits eaten as raw as well as cooked followed by other components. However, leaf and leaf-shoot were used as cooked for vegetable, which was the most applicable consumption mode after fruits. Although, other components such as tubers, young stem-shoots, seeds/pods etc. are minimally used.

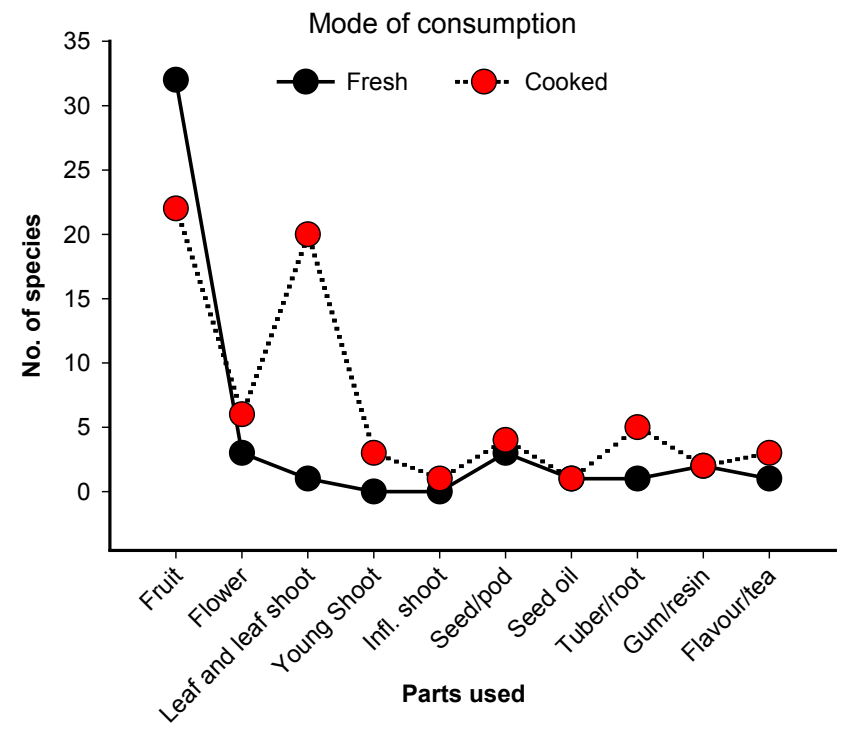

Figure 6. Component-wise consumption mode of edible plant species 
In case of certain edible plants, conventional and non-conventional processing is essential to make it palatable. For example, shoots of Bamboo, Agave and Thor shoots (Euphorbia royleana) are peeled off or outer hard part is removed, cut into small pieces, then boiled in water to remove it bitterness, then fried and cooked. However, some plants can be directly consumed without processing. Underground parts or tuber require washing, slicing and boiling for particular time to discard the acrid content of the tuber. Leaves of Jangli Kachalu (Colocasia esculenta) are steamed before cooking into vegetable. Flower buds of Kachnaar (Vauhinia variegata) is boiled in water, squeezed off and then cooked into vegetable.

\section{Discussion}

\subsection{Edible Plant Diversity and Its Edibility}

Wild edible plants are the alternative source of food which plays an important role to meet the nutritional requirements of the local people in remote or rural areas of India as well as in the other developing countries of the world. The documentation of traditional knowledge on utilization by the local people of wild plants and its nutritive values is one of the main objectives of this study as the most of traditional information of these plants is still poorly documented. At global level, particularly developing countries, large number of population do not have sufficient food for their daily need. Moreover, the food they consume is deficient of nutrients [24]. The present study indicates the potential of underutilized plants as the source of non-conventional food which can be good source of potential food supplements and cheaper alternative food across the hilly regions [3]. In addition, many of these plants are rich in minerals and vitamins and may act as potential nutraceutical supplements. Consumption of these fruits, vegetables is known to lower the risk of several life style diseases such as cardiovascular disease, cancer, diabetes, stroke etc. ([18], [3]).

Himalayan region is the main storehouse of wild edible plants as it is evident through the studies ([25], [16]). In present study, 85 edible plant species were explored from selected sites. On the basis of plant parts used; out of 85 species, maximum plant species are utilized as fruit (45 species) followed by leaf (13 species), whole aerial part (WAP, 12 species), flower/ inflorescence (9 species), seeds (6 species), tuber (6 Species), stem-shoot (3 species), whole plant (WP, 2 species) and 2 species as gum and one species as resin (Figure. 7). In conformity with this, Sharma et al.[20], reported 25 wild edible plants used as fruit, 22 as leaf and 13 used as root from Mandi district, Himachal Pradesh confirms that edible wild plants have substantial access to search food for future prospect.

Some plants have shown substantial edible quality that might be a good hope for searching new quality-wise edible plants particularly rich with nutritive values. Literature survey indicates that study concerning exploration and documentation of wild edible plants in India is certainly not a new task, it is perhaps linked with scale of pre-historic developmental period. However, the research conducted on such plants is not comprehensive and integrative e.g. Sehgal and Sood, [26] reported nutritional components of only one wild edible fruit (Ziziphus jujuba Mill.) and moreover some studies have been reported on medicinal, traditional and ethno botanical aspects but not focused on diversity and status of underutilized edible plants in the same region. Kumar and Choyal [27] recorded 16 plants traditionally used for curing snake bites by the Local rural people and ethno botanical notes of 26 plant species[28]; 91 plant species used for curing different ailments by Sharma et al.,[20] and Devi et al.,[29], recorded 20 plants from Fabaceae and Solanaceae families for ethno-medicinal purposes.

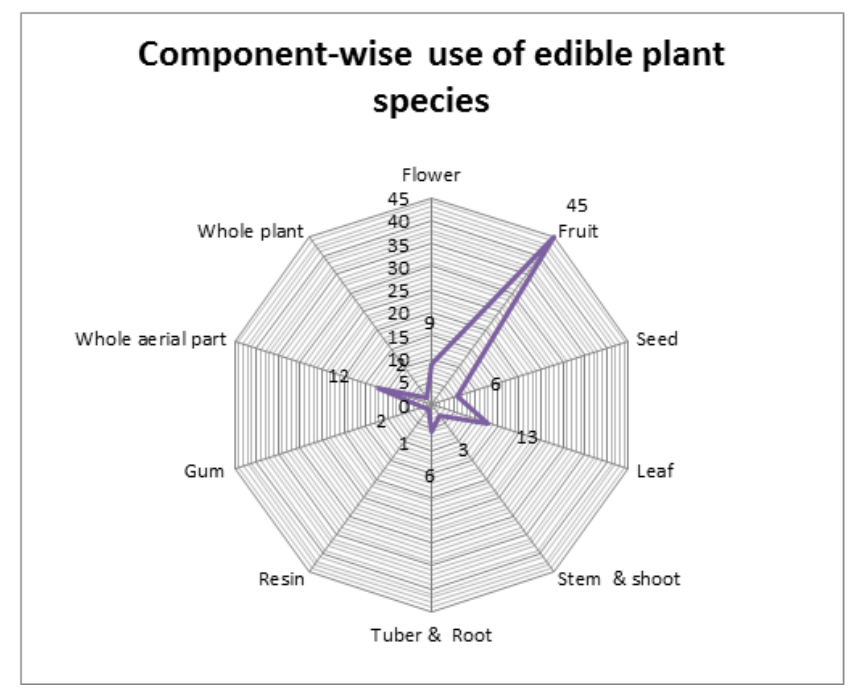

Figure 7. Component-wise use of edible plant species

Species documented in present study are of various life forms, of which, majority of species are trees followed by herbs, creepers and shrubs. Family-wise distribution of the recorded plants revealed 39 families; of which, highest number of species belong to family Moraceae (11) followed by Cucurbitaceae (8) Fabaceae (6), Amaranthaceae and Rosaceae (5), Many families (14 in total) had 2 to 4 species and remaining other 20 families have minimum no. of one species. In conformity with this, 9 species of Moraceae family were reported by Sharma and Mishra [22], (study was conducted at Bilaspur district in Himachal Pradesh).

It is widely accepted that traditional knowledge is very valuable resource which may provide useful leads for scientific research [21]. Most of wild edible or underutilized plants traditionally consumed either as raw fruits, cooked leafy and tuber vegetables having nutritive rich elements diets which can compensate dietary deficiency of nutrients and vitamins necessary for human [30]. In the present study, it was observed that consumption mode of various plant parts were used as mainly cooked or as fresh; of which, maximum numbers of wild plants as fruits are generally eaten raw when they ripe and also unripe fruits, seeds, flowers and leaves are used as cooked in form of vegetables (described in Figure 6). Across component-wise usage, highest number of species 
(45) was used as fruits followed by other components like leaf (13), whole aerial part (12), flower (9), seed (6), tuber and root (6), gum \& resin (4) stem \&shoot (3), and whole plant (2), respectively (Figure. 7). In this study, however, consumption of leaf and leaf shoot as cooked vegetable was major mode of consumption after fruits. Sharma et al. [20], who conducted a study on wild edible plants in Mandi district, Himachal Pradesh, also reported that maximum species ( $25 \mathrm{spp}$.) were used as fruits followed by leaves ( 22 spp.) and roots (13 spp.). Based on general perceptions of the local people, fruits are indeed rich source of protein and energy; therefore it can be used to combat malnutrition [31]. However, after fruits and leaf, other parts like tubers, young shoots and inflorescence shoots etc. are less consumed e.g. Dioscorea bulbifera, D. deltoidea, and Pueraria montana used as tuber, and Euphorbia royleana, Bambusa vulgaris and Agave amaricana as young stem and young inflorescence whereas, Achyranthes aspera, Amaranthus cruentus, Bauhinia vahlii, Pinus roxburghii, Celastrus paniculatus as seed and Butea monosperma, Cordia dichotoma, Cordia sinensis and Barberis asiatica are used as gum \& resin. In addition to this, young pods of Cassia fistula is used to prepare pickle and Murabba (a preparation of sweet jelly) and young shoots of Euphorbia royleana are being used to prepare vegetable by local people; this is perhaps a new kind of use not reported in literature. It is noted that some plants are commonly available throughout the region, therefore, use and applications of following species for food purposes are more harnessed by local people, such as: Aegle marmelos, Bauhinia variegata, Cordia dichotoma, Ficus carica, Ficus palmata, Phyllanthus emblica, Pyrus pashia, Syzygium cuminii, Berberis asiatica, Punica granatum, Morus alba, Murraya koenigii, Colocasia esculenta, Amaranthus viridis, Amorphophallus paeoniifolius, Centella asiatica, Chenopodium album, Digera muricata, Ocimum basilicum, Viola serpense, Celastrus paniculatus, Dioscorea deltoidea, Momordica dioica etc. Due to several reasons, the use of some plants is day by day declining; however, more specifically it was observed that local people are changing traditional living style and adopting modern lifestyle rapidly, therefore, exploration regarding edibility and nutrient components of the wild plants is an urgent need of the hour. Furthermore, which can be a basis to solve the problem malnutrition especially in developing countries where malnutrition and food security are main issues [32]. Moreover, conservation and harnessing of those useful wild species can be taken for future prospects.

\subsection{Role of Edible Plant Species on Human Health Issues}

In general, those wild plant species have been reported across tropics or any habitat from the globe having certain amount of medicinal sense. Since wild edible plants have played an important role in human life since time immemorial particularly in the rural and tribal communities, who depends upon wild food resources to get food and remedies of ailments. Therefore, it is again pressure on tribal communities who are residing in remote, mountainous and adjacent to the forest areas where no proper facilities of transportation and any kind of health care system is available. Hence, local and tribal communities are bound to search wild flora for food and medicinal purposes.

However, main objective of our present study was to explore edibility assessment of the underutilized wild flora because information concerned to this is substantially lacking while a significant number of documents are found in literature on ethno medicinal and ethno-botanical purposes, therefore, we found medicinal uses of almost all recorded species in the literature. Details are given in the Table 3. Many of the edible wild plants that are included in local food baskets have both therapeutic (medicinal) and dietary functions. In addition, several wild edible plant species having rich macro and micronutrients qualities with antimicrobial and antioxidant properties, which are essential to check diseases and for better health. From a nutritional viewpoint, it is important to pay attention to this group of traditional foods for several reasons. Their direct nutritional contribution is often significant but neglected and not well understood. 
Table 3. Potential role of wild edible plants on Human health related problems and source of minerals enrichment. Present information of recorded wild edible plants assembled from literature is given in this table.

\begin{tabular}{|c|c|c|c|}
\hline $\begin{array}{l}\text { Sr. } \\
\text { no }\end{array}$ & Plant Species & Diseases & Source \\
\hline 1 & Aegle marmelos & $\begin{array}{l}\text { Abdominal pain, Constipation, Asthma and Sexual debility, } \\
\text { Dysentery, Vitamin C. }\end{array}$ & ([34], [35], ([36], [37]). \\
\hline 2 & Achyranthes aspera & $\begin{array}{l}\text { Pneumonia, Weakness, Fever, Cough and Cardiac tonic, Hydrophobia, } \\
\text { Diuretic and Abortion }\end{array}$ & ([34], [38], [35], [39]). \\
\hline 3 & Aloe vera & Jaundice, Stomach problems and Burning sensation & [39]. \\
\hline 4 & Amaranthus spinosus & Boils, Snake bite, Toothache, Piles and Iron, Zinc rich & $([38],[40])$. \\
\hline 5 & Amaranthus viridis & $\begin{array}{l}\text { Constipation, Piles, Bronchial problems, Eye vision, Cooling, } \\
\text { Laxative, Leucorrhoea, Snake bite, Antipyretic, Leprosy, Burning } \\
\text { sensation and Iron, Zinc rich }\end{array}$ & {$[40]$} \\
\hline 6 & Artemisia scoparia & Burns & [38] \\
\hline 7 & Bauhinia variegata & Abdominal tumor and rich in Potassium and Calcium & $([37],[40])$ \\
\hline 8 & Berberis asiatica & $\begin{array}{l}\text { Blood purifier, Cooling, Laxative, Diaphoretic, Stomach, Diarrhoea, } \\
\text { Piles, Eye infection, Fever, Ulcer, Skin diseases, Rheumatism, } \\
\text { enlargement of spleen, Cholera and Dyspepsia }\end{array}$ & [39] \\
\hline 9 & Boerhavia diffusa & $\begin{array}{l}\text { Eye swelling and watering of eyes, Jaundice, Night blindness, } \\
\text { Eczema, Rheumatism and Abortifacient }\end{array}$ & ([34], [35], [38]) \\
\hline 10 & Bombax ceiba & $\begin{array}{l}\text { Skin diseases, Pimples, Leucorrhoea, Menstrual disorder, Urinary } \\
\text { problems and Tonic }\end{array}$ & $([35],[36],[37])$ \\
\hline 11 & Butea monosperma & $\begin{array}{l}\text { Cuts, Wounds, Swelling, Bone fracture, Leucorrhoea and Bleeding at } \\
\text { the birth of child }\end{array}$ & ([34],[35],[37] \\
\hline 12 & Carissa spinarum & Constipation, Indigestion and Appetizer & [41] \\
\hline 13 & Cassia fistula & $\begin{array}{l}\text { Paralysis, Rheumatism, Jaundice, Wound, Eczema, Headache and } \\
\text { Acidity }\end{array}$ & ([37], [42]). \\
\hline 14 & Celastrus paniculatus & Rheumatism Increase memory and Headache & $([35],[36])$. \\
\hline 15 & Centella asiatica & Increases memory, Fever and Cardiac problem & $([36],[42])$ \\
\hline 16 & Chenopodium album & Stomach pain Swollen gum and Zinc rich & ([36], [38], [40]). \\
\hline 17 & Coccinia grandis & Cough, cold, Diabetes and Throat infection & ([36], [37]). \\
\hline 18 & Colocasia esculenta & Atrophy, Cough and Bronchial disorder & [37] \\
\hline 19 & Commelina benghalensis & $\begin{array}{l}\text { Leprosy and epistaxis, Epilepsy, Stomach disorder, Eye sore, Treating } \\
\text { sterility Throat sore and Calcium rich }\end{array}$ & ([37], [40]). \\
\hline 20 & Cordia sinensis & Mouth ulcer and Indigestion & ([35], [37]). \\
\hline 21 & Cordia dichotoma & Mouth ulcer and Indigestion & $([35],[37])$. \\
\hline 22 & Digera muricata & Boils & [34] \\
\hline 23 & Dioscorea bulbifera & $\begin{array}{l}\text { Intestinal Worms, Abdominal Pain, Boils, Bone fracture, Piles, } \\
\text { Dysentery Calcium and Potassium rich }\end{array}$ & ([35], [37], [40]). \\
\hline 24 & Dioscorea deltoidea & Dysentery Piles, Calcium and Potassium rich & ([43], [40]). \\
\hline 25 & Diospyros melanoxylon & Urinary and Heart diseases and Antidysentric & {$[37]$} \\
\hline 26 & Duchesnea indica & Cuts, Eye diseases, Swelling and Ulcer & [43] \\
\hline 27 & Euphorbia thymifolia & Skin diseases, Bone fracture and Eye problems & [43] \\
\hline 28 & Ficus auriculata & Cause infertility & [37] \\
\hline 29 & Ficus benghalensis & $\begin{array}{l}\text { Piles, Boils and blisters, Spermatorrhoea, Snake bite and Sexual } \\
\text { potentiality }\end{array}$ & $([38],[37])$. \\
\hline 30 & Ficus carica & $\begin{array}{l}\text { Boils, Eruption, Skin disease, Gastric problems, Cancer, Wart, } \\
\text { Inflammation and Calcium rich }\end{array}$ & ([37], [40]). \\
\hline 31 & Ficus glomerata & Diarrhoea, Bleeding piles and Acidity & [44] \\
\hline 32 & Ficus hispida & Leucoderma & {$[37]$} \\
\hline 33 & Ficus palmata & Constipation, Lung, Bladder diseases; Calcium rich & ([40], [45]). \\
\hline 34 & Ficus religiosa & $\begin{array}{l}\text { Ulcers, Asthma, Fistula of annus, Prevent conception, Skin diseases } \\
\text { and Abortifacient }\end{array}$ & $([38],[36])$. \\
\hline 35 & Flacourtia indica & Antidote & [37] \\
\hline 36 & Fragaria vesca & Menstrual problems, Laxative and Purgative & [41] \\
\hline 37 & Luffa operculata & Jaundice & {$[37]$} \\
\hline 38 & Momordica dioica & Blood purifier, Diabetes and Eye infection & [37] \\
\hline
\end{tabular}




\begin{tabular}{|c|c|c|c|}
\hline 39 & Morus alba & Sore throat, Dyspepsia, Anthelmintic, Purgative and Vermifuge & [41] \\
\hline 40 & Mucuna prurarium & $\begin{array}{l}\text { Mental and renal disorder, Snake bite, Diuretic, Constipation, } \\
\text { Purgative and Aphrodisiac }\end{array}$ & ([43], [44]). \\
\hline 41 & Murraya koenigii & $\begin{array}{l}\text { Dysentery, Diarrhoea, Malarial fever, Tonic, lactation and } \\
\text { Anthelmintic }\end{array}$ & [41] \\
\hline 42 & Ocimum basilicum & Increases potency in men & [37] \\
\hline 43 & Oxalis corniculata & Skin diseases, Toothache, Piles, Anemia and Calcium rich & $([42],[44,[40])$. \\
\hline 44 & Oxalis rubra & Dysentery, Fever, Insect bite, skin disease and Stomach problem & [43] \\
\hline 45 & Phoenix sylvestris & Heartburn, Loss of consciousness and vertigo & [37] \\
\hline 46 & Phyllanthus emblica & $\begin{array}{l}\text { Constipation, Dysentery, Leucorrhoea, Stop bleeding, Hypertension } \\
\text { and Vitamin C rich }\end{array}$ & ([34], [38], [40]). \\
\hline 47 & Physalis peruviana & Earache, Spleen disorder, Gastric and Stomachache & $([36],[44])$. \\
\hline 48 & Pinus roxburghii & Cuts, Wounds, Antibacterial and Cracks on feet & [45] \\
\hline 49 & Portulaca oleracea & $\begin{array}{l}\text { Cardiac stimulant, Skin burning, Sores, Cough, Burn, Earache, } \\
\text { Febrifuge, Diuretic }\end{array}$ & [40] \\
\hline 50 & Pueraria montana & $\begin{array}{l}\text { Abdominal pain, antiemetic, Asthma, Body ache, Cholera and } \\
\text { Diarrhoea }\end{array}$ & [37] \\
\hline 51 & Punica granatum & $\begin{array}{l}\text { Cough, Cold, Cardiac tonic, Bleeding nose, Brain affection, } \\
\text { Dysentery, Diarrhoea, Worms and Source of Vitamin C }\end{array}$ & [39] \\
\hline 52 & Pyrus pashia & Swelling and Cuts & [43] \\
\hline 53 & Rosa moschata & Eye troubles, Joint pain, Diarrhoea, Ophthalmia and Wounds & ([39], [43]). \\
\hline 54 & Rubus ellipticus & Laxative & [39] \\
\hline 55 & Rumex hastatus & $\begin{array}{l}\text { Stomachic, Astringent, Carminative, Dysentery, Jaundice and } \\
\text { Calcium rich }\end{array}$ & $([40],[41])$. \\
\hline 56 & Solanum nigrum & $\begin{array}{l}\text { Liver Complaints, Blood purifier, Antidote, Boils, Antiemetic, } \\
\text { Diarrhoea, Eye infection and Hydrophobia }\end{array}$ & $([37],[40])$ \\
\hline 57 & Spilanthes acmella & $\begin{array}{l}\text { Mouth and throat affection, Tongue paralysis and Stammering in } \\
\text { Children }\end{array}$ & [39] \\
\hline 58 & Syzygium cuminii & $\begin{array}{l}\text { Diabetes, Anti dysentery, Headache, Increase appetite, Blisters in } \\
\text { mouth, Cancerous sore, Carminative, Diuretic }\end{array}$ & ([37], [44]). \\
\hline 59 & Terminalia bellirica & $\begin{array}{l}\text { Asthma, Cold \& Cough, Leprosy, Liver complaint, Stomach problems, } \\
\text { Piles, Liver, Fever. Rheumatism and Purgative }\end{array}$ & ([37], [44]). \\
\hline 60 & Terminalia chebula & Body heat & [42] \\
\hline 61 & $\begin{array}{l}\text { Trichosanthes } \\
\text { cucumerina } \\
\text { cucumerina }\end{array}$ & Bronchial problems and Heart diseases & [37] \\
\hline 62 & Viola canescens & Cough, cold, Asthma, Bronchial, Eye problems and Asthma & ([39], [43]). \\
\hline 63 & Woodfordia fruticosa & $\begin{array}{l}\text { Diarrhoea, Asthma, Boils, Rheumatism, Tonic in weakness in } \\
\text { pregnancy, Fever, Night blindness, Cough, Dysentery and Cholera }\end{array}$ & ([37], [43], [44]). \\
\hline 64 & Ziziphus mauritiana & Fever, Blood purifier, Diarrhoea and Digestive disorder & [37] \\
\hline
\end{tabular}

Due to insufficient scientific evidences on about health benefits of regular consumption of small quantities of medicinal foods, many of important medicinal plant species from wild regions are still waiting for proper diagnosis and investigation. According to Abbasi et al. [40], the traditional plant medicines may be the contribution of small quantities of trace minerals and vitamins. Further, they expressed that wild plants are found in uncultivated land, such as forests, which have not been depleted of minerals, and also act as alternative source of micro-nutrients for cultivated crops [46]. Many studies reveled that consumption of wild edible plants might be an important mediator for curing different disease and may be fed as food supplements to chalk out and fill the gap of deficient food in mineral and micro-nutrients([34],[35], [36], [40]). Wild edible plants could prove clinically useful for improving the health and disease outcomes among the food insecure. Documentation of these plant species, traditional uses, nutritive and medicinal properties, ecological status could benefit the health of human and animals in the societies. The current research suggests that wild edible plants should be considered in studies examining health concerns in food insecure populations.

\section{Conclusions}

The existing observations indicate that the local people of Hamirpur district of Himachal Pradesh, India who are particularly living in remote and high altitude areas mainly rely upon the plant resources adjacent to their surroundings to meet their day-to-day requirements. But today new generation is continuously forgetting the use of local recourses as food and depends upon staple food plants only. To replace the existing burden on staple crops to meet the need of whole population, there is an urgent need to explore 
other alternative food plants having rich potential and excellent source of energy. For this, nutritional prospects of the plants need to be worked out which can help to overcome the nutritional deficiencies especially in remote areas.

In conclusion, in this study, we found that maximum numbers of wild edible plants are used as fruit and leaf followed by other components. Generally, fruits are eaten as raw when they ripe and usually unripe fruits, seeds, flowers and leaves are cooked as vegetables. Majority of edible plants do not require processing whereas some plants need to be processed. Therefore, there is also a need to find out the traditional method of food processing and status of nutrients after processing in addition to documentation and exploration of wild plants for better edibility, investigation for their role in maintaining aesthetic ecosystem and environment for local people. The Food and Agricultural organization (FAO) already recognizes that 'nutrition and biodiversity converge to a common path leading to food security and sustainable development 'can play as a key role in global nutrition security' [5].

\section{Competing Interests}

Authors declare that they have no competing interests

\section{Authors' Contributions}

ANS designed experimental establishment, RCB performed field study and drafted manuscript, $\mathrm{CN}$ helped in preparation and finalized manuscript. All authors have equal role in preparation and finalizing the manuscript.

\section{Acknowledgement}

We are very thankful to the local people and administration of Hamirpur district Himachal Pradesh for providing necessary facilities and kind cooperation required during field work. Thanks are due to Chairperson, Department of Botany, Panjab University Chandigarh provided for required facilities during course of work. First author is gratefully acknowledged to University Grants Commission, Government of India, New Delhi for financial support in form of Teacher Fellowship Award and Department of Higher Education, Haryana for granting study leave for doctoral work.

\section{REFERENCES}

[1] Sharma P K, Chauhan N S and Lal B, Study on plants associated indigenous knowledge among the Malanis of Kulu district, Himachal Pradesh, Indian Journal of Traditional Knowledge, 4(4): 403-408, 2005.
[2] Tebkew M, Zebene Asfaw Z and Zewudie S, Underutilized wild edible plants in the Chilga District, northwestern Ethiopia: focus on wild woody plants, Agriculture \& Food Security, 3: 12, 2014.

[3] Pal R S, Kumar R A, Kant L and Bhatt J C, Wild edible potential nutraceutical fruit in Indian Himalayan region, Popular Kheti, 2(3): 199-203, 2014.

[4] Abbasi A M, Khan M A, Shah M H, Shah M M, Pervez A and Ahmad A, Ethno botanical appraisal and cultural values of medicinally important wild edible vegetables of lesser Himalayas, Pakistan Journal of Ethno biology and Ethno Medicine, 9:1-13, 2013.

[5] FAO, How to feed the world in 2050, Food and Agriculture Organization of United Nations, Rome, Italy, 1-35, 2009.

[6] Godfray H C J, Bedding J R, Crute I R, Hudded L, Lawrence D, Muir J F, Pretty J, Robinson S, Thomas S M and Toulmin C, Food Security: The challenges of feeding 9 billion people, Science, 327: 812-818, 2010.

[7] Misra S and Misra M K, Ethnobotanical study of plants with edible underground parts of south Odisha, India, International Journal of Agricultural and Food Science, 4(2):51-58, 2014.

[8] Kumar D, Dwivedi S V, Kumar S, Ahmed F, Bhardwaj R K, Thakur K S and Thakur P, Potential and biodiversity conservation strategies of underutilized or indigenous vegetables in Himachal Pradesh, International Journal of Agricultural Sciences, 10:459-462, 2014.

[9] International Plant Genetic Resources Institute (IPGRI), Monograph on Underutilized Crops, Rome, Italy IPGRI, Enhancing the contribution of neglected and underutilized Species to Food Security and Income of Rural Poor, 2001. (http:www.ipgri.cgiar.org).

[10] International Plant Genetic Resources Institute (IPGRI), Neglected and underutilized plant species: Strategic action plan of the International Plant Genetic Resources Institute Rome, Italy, 2002.

[11] Aboagye L M, Obirih-Opareh N, Amissah L and Adu-Dapah $\mathrm{H}$, Underutilized species, policies and strategies, Analysis of existing national policies and legislation that enable or inhibit the wider use of underutilized plant species for food and agriculture in Ghana, Council for Scientific and Industrial Research, Ghana. 2007.

[12] Nirmala C, Bisht M S and Sheena H, Nutritional properties of bamboo shoot: potential and prospects for utilization as a health food, Comprehensive Reviews in Food Science and Food Safety, 10:153-165, 2011.

[13] Nirmala C, Bisht M S and Laishram M, Bioactive compounds in bamboo shoot: health benefits and prospects for developing functional foods, International Journal of Food Science and Technology, 49:1425-1431, 2014.

[14] Kaval I, Behcit L and Cakilcioglu U, Survey of wild food plants for human consumption in Gecitli, Indian Journal of Traditional Knowledge, 14(2):183-190, 2015.

[15] Mekbib Y and Derresa T, Exploration and collection of root and tuber crops in east Wollega and Ilu Abobora Zone: Rescuing declining genetic resources, Indian Journal of Traditional Knowledge, 15(1):86-92, 2016.

[16] Reddy K N, Pattanaik C, Reddy C S and Raju V S L, Traditional knowledge on wild food plants in Andhra Pradesh, Indian Journal of Traditional Knowledge, 6(1): 223-229, 2007. 
[17] Bandyopadhyay S and Mukherjee S K, Wild edible plants of Koch Bihar district, West Bengal, Natural Product Radiance, 8(1):64-72, 2009.

[18] Chauhan P P, Nigam A and Santvan V K, Ethnobotanical survey of trees in Pabbar Valley, Distt. Shimla, Himachal Pradesh, Life Sciences Leaflets, 52:24-39, 2014.

[19] Chowdhery H J and Wadhwa BM, Flora of Himachal Pradesh, Botanical Survey of India, Vol. 1, Vol. 2 and Vol. 3, 1984

[20] Rana P K, Kumar P, Singhal V K and Rana J C, Uses of local biodiversity among the tribal communities of Pangi Valley of district Chamba in cold desert Himalaya, The Scientific World Journal, 1-15, 2014.

[21] Sharma P, Agnihotry A, Sharma P P and Sharma L, Wild edibles of Murari Devi and surrounding areas in Mandi district of Himachal Pradesh, India, International Journal of Biodiversity and Conservation, 5(9): 592-604, 2013.

[22] Sharma M and Sood S K, Ethnobotanical survey of wild plant of district Solan, Himachal Pradesh, India, International Journal of Environmental Biology, 3(3): 87-95, 2013.

[23] Sharma P and Mishra N K, Diversity, utilization pattern and indigenous uses of plants in and around a cement factory in Bilaspur district of Himachal Pradesh, North-Western Himalaya, Biological Forum, 1(2): 89-9, 2009.

[24] Kharwal A D and Rawat D S, Ethnobotanical study and distribution of different Rhododendron species in Himachal Pradesh, Plant Science Feed, 3(3):46-49, 2011.

[25] Sharma P K, Thakur R, Gulshan, Deepika and Kumar D, Studies on some important medicinal and aromatic plants and their traditional usages in district Hamirpur -A sub Himalayan tropical region of Himachal Pradesh- India, Weekly Science Research Journal, 1(7): 2321-7871, 2013.

[26] Samant S S and Dhar U, Diversity, endemism and economic potential of wild edible plants of Indian Himalaya, International Journal of Sustainable Development and World Ecology, 4: 179-191, 1997.

[27] Sehgal A B and Sood S K, Nutritional analysis of edible wild fruit (Zizyphus jujubaMill.) used by rural populace of district Hamirpur, (H.P.), India, IOSR- Journal of Pharmacy and Biological Sciences, 6(2): 46-49, 2013.

[28] Kumar N and Choyal R, Traditional phytotherapy for snake bites by the local rural people of Hamirpur district in Himachal Pradesh (India), Biological Forum - An International Journal, 4(1): 98-106, 2012.

[29] Kumar N and Choyal R, Ethnobotanical notes on some plants of Hamirpur district of Himachal Pradesh used in the treatment of arthritis, rheumatism and other inflammatory disorder, Indian Journal of Plant Science, 1 (2\&3):1-8 ,2014.

[30] Devi S, Gupta A K and Singh M, Ethano-Medicinal use of Plants Belonging to Families Fabaceae and Solanaceae, Hamirpur District (H.P.), International Journal of Scientific and Research Publications, 2(11):1-4, 2012.

[31] Buragohain J, Singh V B, Deka B C, Jha A K, Wanshnong K and Angami T, Collection and evaluation of some underutilized leafy vegetables of Meghalaya, Indian Journal of Hill Farming, 26(2):111-115, 2013
[32] Rathore M, Nutrient content of important fruit trees from acrid zone of Rajasthan, Journal of Horticulture and Forestry, 1(7): 103-108, 2009.

[33] Rastogi A and Shukla S, Amaranth: A new millennium crop of nutraceutical values, Reviews in Food Science and Nutrition, 53:109-125, 2013.

[34] Katewa SS, Chaudhary BL and Jain A, Folk herbal medicines from tribal area of Rajasthan, India, Journal of Ethno-pharmacology, 92:41-46, 2004.

[35] Kosalge SB and Fursule RA, Investigation of ethno-medicinal claims of some plants used by tribals of Satpuda Hills in India, Journal of Ethno-pharmacology, 121: 456-461, 2009.

[36] Jain A, Katewa SS, Chaudhary DL and Galav P, Folk herbal medicines used in birth control and sexual diseases by tribals of southern Rajasthan, India, Journal of Ethno-pharmacology, 90: 171-177, 2004.

[37] Jain A, Katewa PK, Galav PK and Sharma P, Medicinal plant diversity of Sitamata wildlife sanctuary, Rajasthan , India, Journal of Ethno-pharmacology, 102: 143-157, 2005.

[38] Parveen, Upadhyay B, Roy S, Kumar A, Traditional uses of medicinal plants among the rural communities of Churu district in the Thar Desert, India, Journal of Ehtno-pharmacology, 113:387-399, 2007.

[39] Singh KJ and Thakur AK, Medicinal plants of the Shimla hills, Himachal Pradesh: A Survey, International Journal of Herbal Medicine, 2(2): 118-127, 2014.

[40] Abbasi A M, Shah M H and Khan M A, Wild Edible Vegetables of Lesser Himalayas, Ethno-botanical and Nutraceutical Aspects, Volume 1, Springer Cham Heidelberg New York London, 2015. Springer International Publishing Switzerland 2015

[41] Sharma P, Agnihotry A, Sharma PP and Sharma PP, Wild edibles of Murari Devi and surrounding areas in Mandi district of Himachal Pradesh, India, International Journal of Biodiversity and Conservation, 5(9): 592-604, 2013.

[42] Rajakumar N and Shivanna MB, Ethno-medicine application of plants in eastern region of Shimoga district, Karnataka, India, Journal of Ethno-pharmacology, 126: 64-73, 2009.

[43] Pooja P, Pankaj S and Ajit A, Diversity, Utilization pattern and indigenous uses of floristic diversity in Murari Devi and surrounding areas of Mandi district, Himachal Pradesh (India), Journal of Biodiversity and Ecological Sciences 2(3): 196-216, 2012

[44] Poonam K and Singh GS, Ethno-botanical study of medicinal plants used by the Taungya community in Terai Arc Landscape , India, Journal of Ethno-pharmacology, 123: 167-176, 2009.

[45] Chauhan PP, Nigam A and Santvan VK, Ethno-botanical survey of trees in Pabbar Valley, Distt. Shimla, Himachal Pradesh, Life Science Leaflets, 52: 24-39, 2014.

[46] Ogle BM, Tuyet HT, Duyet HN and Dung NNX, Food, Feed or Medicine: The multiple functions edible wild plants in Vietnam, Economic Botany, 57(1): 103-117, 2003. 